\title{
Proposta do Uso de Análise de Sentimento no Desenvolvimento de uma nova Métrica de Sustentabilidade
}

\section{Alternative Title: Proposal Sentiment Analysis Use in Developing a new Sustainability Metrics}

\author{
Nataly Bruna Aires \\ Faculdade Metropolitana de Curitiba \\ Rui Barbosa, 5881 - 83045-350 \\ São José dos Pinhais/PR \\ natalyhundert@gmail.com
}

Pietra Sales

Faculdade Metropolitana de Curitiba

Av. Rui Barbosa, 5881 - Afonso Pena

83045-350 - São José dos Pinhais/PR pietrasalles300@gmail.com

\author{
Lucas Vieira Lopes \\ Faculdade Metropolitana de Curitiba \\ Rui Barbosa, 5881 - 83045-350 \\ São José dos Pinhais/PR \\ lucasv.lopes@hotmail.com
}

Marcos van Vessen $\mathrm{Jr}$

Faculdade Metropolitana de Curitiba

Rui Barbosa, 5881 - 83045-350

São José dos Pinhais/PR

marcos.vessen@ielpr.org.br

\author{
Eduardo F. Machado \\ Faculdade Metropolitana de Curitiba \\ Rui Barbosa, 5881 - Afonso Pena \\ 83045-350 - São José dos Pinhais/PR \\ eduardo_fe_machado@yahoo.com.br \\ Cassiana F. da Silva \\ Faculdade Metropolitana de Curitiba \\ Av. Rui Barbosa, 5881 - Afonso Pena \\ 83045-350 - São José dos Pinhais/PR \\ cassiana.silva@ielpr.org.br
}

\begin{abstract}
RESUMO
Métricas de Sustentabilidade são medidas e indicadores que visam servir como ferramentas de gestão, tanto no nível macro, para políticas públicas, por exemplo, quanto no micro, na eficiente gestão de uma empresa. Com seu uso pode-se mensurar os impactos das práticas sustentáveis, quanto à correlação, por exemplo, entre aumento de receita e da participação de mercado, com práticas tais como: redução de despesas com energia, redução de despesas com resíduos, redução de despesas com materiais e água. Ou ainda a correlação entre um ambiente onde se promove a Sustentabilidade e o aumento de produtividade dos funcionários, redução de gastos com contratações e perda de pessoal. Indiscutivelmente se faz necessário um alinhamento cada vez maior entre o discurso e a prática dos valores da Sustentabilidade. Neste contexto, o presente artigo tem como propósito utilizar a Análise de Sentimentos no desenvolvimento de uma nova métrica. No estágio atual, foi aplicado um questionário a respeito da opinião dos funcionários da Instituição de Ensino Superior FAMEC - PR quanto à sustentabilidade organizacional. As respostas do questionário passaram por análise, por meio da ferramenta RapidMiner, onde os sentimentos dos funcionários puderam ser qualificados em positivo, negativo ou neutro.
\end{abstract}

\section{Palavras-Chave}

Métricas de Sustentabilidade, Análise de Sentimento.

Permission to make digital or hard copies of all or part of this work for personal or classroom use is granted without fee provided that copies are not made or distributed for profit or commercial advantage and that copies bear this notice and the full citation on the first page. To copy otherwise, or republish, to post on servers or to redistribute to lists, requires prior specific permission and/or a fee.

SBSI 2015, May 26-29, 2015, Goiânia, Goiás, Brazil.

Copyright SBC 2015.

\begin{abstract}
Sustainability metrics are measures and indicators that are intended to serve as management tools, both at the macro level, to public policy, for example, as in the micro, in the efficient management of a company. With its use one can measure the impacts of sustainable practices, through the correlation, for example, from increased revenue and market share, with practices such as reducing energy costs, reducing expenses with waste reduction costs of materials and water. Or even the correlation between an environment where Sustainability is promoted and the increased employee productivity and reduced spending on hiring. Arguably a growing alignment is needed between the discourse and practice of sustainability values. In this context, this article aims to use the Analysis of Feelings in the development of a new metric. In the present stage was done a survey, to know about the opinion that the Superior Educational Institute FAMEC - PR's workers have about organizational sustentability. The answers passed by an analysis, done with the tool RapidMiner, where the workers feelings were qualified as positive, negative or neutral.
\end{abstract}

\section{Categories and Subject Descriptors \\ I.2.7 [Natural Language Processing]: Text analysis}

\section{General Terms}

Measurement, Experimentation, Human Factors.

\section{Keywords}

Sustainability metrics, Sentiment analysis.

\section{INTRODUÇÃO}

A interdependência constatável das esferas sociais, ambientais e econômicas requer compreensão histórica da relação homemnatureza. O conceito Triple Bottom Line, ou Tripé da Sustentabilidade, sintetizou as inextricáveis características de um 
modelo socialmente justo, ecologicamente correto e economicamente viável, como salienta Veiga [16].

A sustentabilidade, embora seja um tema de grande enfoque na atualidade, não deve ser compreendida como um fenômeno recente. $\mathrm{O}$ conceito de sustentabilidade advém de uma série de evoluções epistemológicas ao longo da história, como explica Leff [9], onde o homem alterou as condições ambientais severamente, comprometendo o equilíbrio espontâneo da natureza. A maneira insustentável dos homens, oriundo de suas organizações e sistemas, foi acentuada após a Revolução Industrial, onde houve a aceleração de exploração em larga escala dos recursos naturais, gerando grande volume de descarte de resíduos no pós-consumo [5]. O contraponto a esse modelo insustentável deu-se por meio da definição do Desenvolvimento Sustentável, do Relatório de Brundtland, em 1987, realizado pela Organização das Nações Unidas (ONU), sendo sua ideia principal a preocupação diante dos recursos disponíveis, de modo a garantir a viabilidade de vida na terra para as gerações vindouras, segundo Comissão Mundial Sobre o Meio Ambiente e Desenvolvimento [3].

A partir de relatórios desenvolvidos em Conferências da ONU, a importância global ao dimensionamento da sustentabilidade tornou-se cada vez maior. Uma das principais características de indicadores de sustentabilidade é sua atuação como componente na avaliação no progresso do desenvolvimento sustentável, como salienta Gallopin [1].

A Métrica da Sustentabilidade é uma ferramenta de medição composta por séries de indicadores a nível social, ambiental e econômico de acordo com o contexto abordado e finalidade de mensuração [8].

As empresas, responsáveis em abarcar os fatores de produção, devem ser os principais agentes no desenvolvimento sustentável, corroborando com a importância das ações humanas, principalmente no alinhamento estratégico da empresa para com os funcionários, para a efetividade de realização de práticas sustentáveis [17]. O engajamento dos funcionários é essencial para a sustentabilidade empresarial. [17]

Desse modo, o artigo objetiva-se em apresentar uma visão genérica de definições e motivações humanas, por meio de estudos realizados na área de sustentabilidade, combinado a apresentação de resultados preliminares no uso de Análise de Sentimentos no desenvolvimento de uma nova Métrica de sustentabilidade.

O texto está estruturado em 6 seções. A seção 1 descreve uma breve contextualização sobre o assunto, bem como suas definições. Na seção 2 é descrito os conceitos de métricas de sustentabilidade. A seção 3 descreve o processo de Análise de Sentimento em termos de suas principais etapas: identificação, classificação de polaridade e sumarização. $\mathrm{Na}$ seção 4 é apresentada a metodologia utilizada para realização do trabalho. A seção 5 relata os resultados preliminares obtidos e por fim, a seção 6 apresenta as conclusões, desafios e direções futuras na área.

\section{MÉTRICAS DE SUSTENTABILIDADE}

Os indicadores de sustentabilidade buscam atuar como componentes na avaliação no progresso do desenvolvimento sustentável, como salienta Gallopin [1]. Logo, a Métricas de Sustentabilidade são medidas compostas por uma série de indicadores no que tange a parte social, ambiental e econômica, que atuarão como ferramenta de gestão conforme finalidade e aplicação.

Os indicadores, como salienta Meadows [1] assumem uma característica intuitiva de monitorar e controlar análises em sistemas complexos. Assim como a aplicabilidade de um termômetro, o indicador visa facilitar a identificação de um dado sintoma, com base nas informações e dados graduais préestabelecidos.

A metodologia de uma Métrica de Sustentabilidade é baseada em modelos teóricos que induzam a construção ou incorporação de indicadores já existentes, seja a nível macroeconômico ou microeconômico de acordo com o que se deseja especificamente mensurar. No que diz respeito ao meio macroeconômico, pode-se citar a métrica desenvolvida em "Medidas de bem-estar e sustentabilidade: desenvolvimento de metodologias de análise e selecção de métricas" [6]. Quanto ao meio organizacional, há a métrica de sustentabilidade aplicada ao "Modelo de mensuração de sustentabilidade empresarial: uma aplicação em vinícolas localizadas na Serra Gaúcha" [2]. A construção de métrica específica para determinada comunidade, pode-se destacar a "Métrica da Sustentabilidade na perspectiva da Geografia: aplicação e avaliação do Painel da Sustentabilidade (Dashboard of Sustentability) na Comunidade Quilombola de Mandira" [8].

O desenvolvimento de uma nova Métrica, voltada ao âmbito empresarial, busca exercer a função de medição de impactos positivos ou negativos, nos resultados financeiros de uma empresa ou organização, tais como aumento da receita e de participação no mercado, devido a práticas ligadas a sustentabilidade como: redução de despesas com energia, redução de despesas com resíduos, redução de despesas com materiais e água, aumento da produtividade dos funcionários, redução de gastos com contratações e rotatividade de pessoal além da redução de riscos [17].

Em síntese, o projeto de Métricas da Sustentabilidade com Mineração de Opinião deve garantir a construção de uma ferramenta na qual seja possível identificar os impactos das práticas sustentáveis nos resultados da empresa e ou negócio, atestando o valor das mesmas.

Uma das hipóteses é que o ambiente criado em uma empresa, cujas práticas estão alinhadas aos princípios da Sustentabilidade, irá aumentar a satisfação dos colaboradores, dessa forma, aumentando a produtividade e reduzindo a rotatividade da mão de obra [17].

Desse modo, com o uso da Análise de Sentimentos, busca-se medir primeiramente a percepção dos colaboradores quanto ao ambiente de trabalho, para que, em uma etapa posterior possa-se avaliar a correlação entre as percepções medidas e o alinhamento de cada empresa.

\section{ANÁLISE DE SENTIMENTO}

Análise de Sentimento, também conhecida por Mineração de Opinião, é um ramo da mineração de textos preocupado em classificar textos não por tópicos, e sim pelo sentimento ou opinião contida em determinado documento. Geralmente associado à classificação binária entre sentimentos positivos e negativos, o termo é usado de uma forma mais abrangente para significar o tratamento computacional de opinião, sentimento e subjetividade em textos, segundo Pang e Lee [13].

Tsytsarau e Palpanas [14] define a análise de sentimentos, como uma área recente que integra pesquisas de mineração de dados, 
linguística computacional, recuperação de informações, inteligência artificial, dentre outras.

Para Liu [10] a análise de sentimentos é definida como qualquer estudo feito computacionalmente envolvendo opiniões, sentimentos, avaliações, atitudes, visões, emoções, expressos em forma textual.

Para Liu [11] a identificação do sentimento em um texto pode ser definida conforme sua granularidade, sendo que a decisão do nível está sujeita ao contexto e aplicação. A análise pode ser em nível de documento, sentença e entidade e aspecto.

No nível de documento, a tarefa é classificar se um documento, expressa um sentimento positivo ou negativo. Este nível de granularidade é adequado quando o documento trata de uma única entidade, isto é, um documento que forneça uma opinião sobre um produto em específico. Já o nível de entidade e aspecto foca na opinião expressa, independentemente das formas utilizadas para expressá-la (por exemplo: documentos, sentenças, orações). Dentre a literatura de Análises de Sentimentos ou Mineração de Opinião, destaca-se o minicurso de "mineração de opinião" apresentado no Simpósio Brasileiro de Sistemas de Informação, 2014 [4].

Ainda para Liu [10] a análise de sentimentos tem origens em comum com a linguística computacional, com a qual compartilha problemas e desafios.

\section{METODOLOGIA}

A metodologia contou com pesquisa bibliográfica para o tema sustentabilidade, que como salienta Mascarenhas [12], tem a investigação centrada em conteúdos extraídos de livros, dicionários, enciclopédias e artigos científicos.

A pesquisa descritiva foi utilizada para compreender dada população ou fenômeno a fim possibilitar o apontamento de níveis e índices, que segundo Gil [7], tem por objetivo estudar opiniões, atitudes e crenças de determinada população.

A ferramenta RapidMiner fornece um ambiente visual para realização de processos de data mining. Essa ferramenta disponibiliza um grande conjunto de funcionalidades, chamadas de operadores, que lhe dá certa independência em relação a outras ferramentas, e ainda conta com um conjunto de extensões que fornece uma maior amplitude no que tange a contextos de utilização.

Com o objetivo de verificar a viabilidade da ferramenta para o estudo em questão, foi criado um questionário no qual foi aplicado a 30 colaboradores da Instituição de Ensino Superior FAMEC - PR. No questionário, os colabores responderam questões como: "Quais são os principais fatores que contribuem ou atrapalham o engajamento do funcionário na empresa que você trabalha?"; "Quais são os fatores que contribuem ou atrapalham a produtividade no seu ambiente de trabalho?"; "Qual é a reputação da sua empresa quanto à sustentabilidade? Explique.".

Após esse processo, as questões foram inseridas na ferramenta RapidMiner e os resultados preliminares são apresentados na seção a seguir.

\section{RESULTADOS PRELIMINARES}

Conforme descrito na seção 4, os experimentos foram gerados através de um questionário aberto e fechado. No questionário foi composto por três questões abertas (descritas seção 4) e três questões fechadas, a saber: Gênero: F ou M; Idade (com uma escala entre as faixas etárias de 18 a 70 anos) e o interesse do colaborador sobre o assunto.

O total de respondentes, cujos perfis são colaboradores da Instituição de Ensino Superior FAMEC, foram 30.

A faixa etária dos colaboradores na maioria dos respondentes está entre "36 a 45 anos" (40\%). A faixa etária de "17 a 25 " corresponde a $20 \%$. De "26 a 35", 13\%. De "56 a 55", 23\%. E a faixa etária de " 56 a mais", somente corresponde a $3 \%$.

Os respondentes foram em sua maioria do gênero masculino, com o total de $70 \%$, e de $30 \%$ do gênero feminino. O interesse sobre o tema Sustentabilidade obtiveram $50 \%$ na alternativa "muito interessado", seguido de $43 \%$ de "pouco interessado", minoritariamente de "razoavelmente interessado", com 7\%.

Como ilustra a Figura 1, foram usados vários operadores para identificar os sentimentos dos funcionários em relação às perguntas já citadas nessa seção.

Após o pré-processamento da ferramenta RapidMiner, foi possível treiná-lo de modo a classificar textos inseridos nos quais apresentavam sentimentos positivos ou negativos sobre o tema. Para esse treinamento foi utilizado o algoritmo SVM (Support Vector Machine) que para Shmilovici [15], é um conjunto de métodos para aprendizado supervisionado, que podem ser aplicados para problemas de classificação e regressão.

Como resultado desse aprendizado observou-se que a ferramenta possibilita a análise de sentimento em relação aos documentos, porém a precisão da classificação da opinião dos colaboradores ainda possui algumas falhas, conforme mostra a Figura 2.

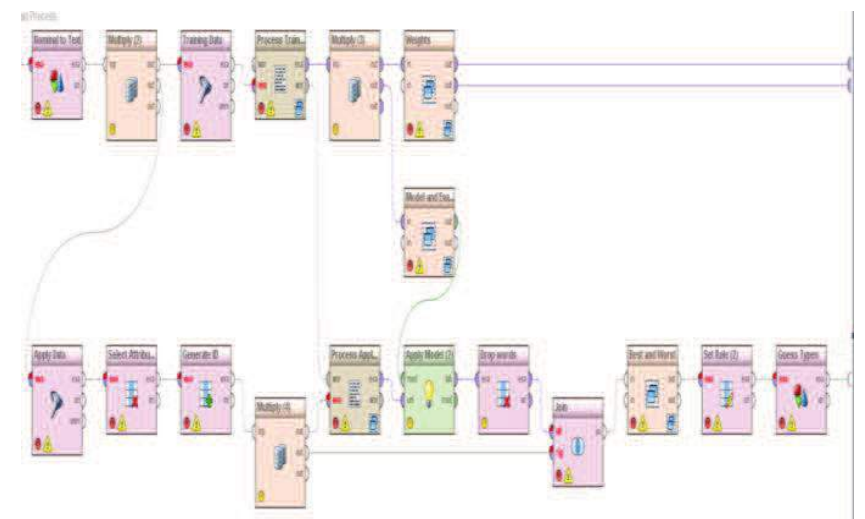

Figura 1. Aplicação das funções de coleta, processamento, aprendizado

Na Figura 2, podemos visualizar todas as repostas já analisadas. Temos na coluna da direita a questão: "Quais são os fatores que contribuem ou atrapalham a produtividade no seu ambiente de trabalho", e na coluna ao lado o resultado da análise de sentimento com a ferramenta RapidMiner.

A Figura 3 apresenta resultados classificados, onde além da análise de sentimento foi feita um score para classificar a validade das respostas com notas de 0 a 100 . 


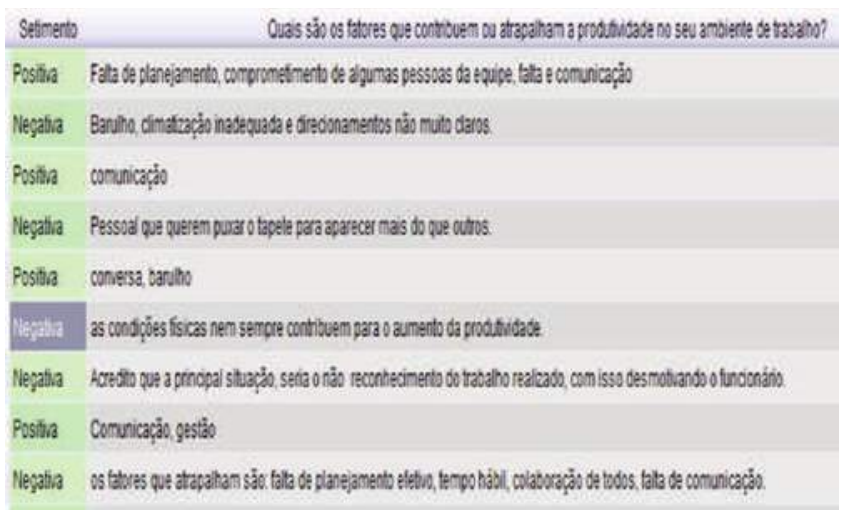

Figura 2. Análise de Sentimentos - Questão 1

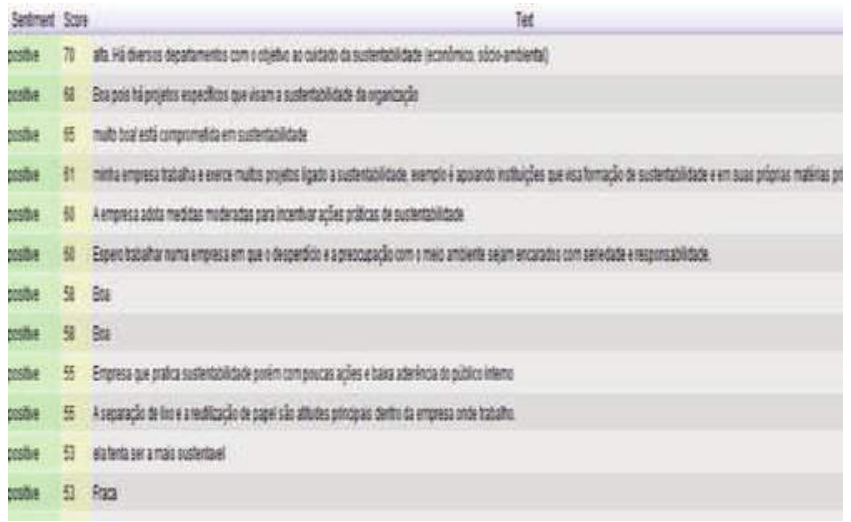

Figura 3. Análise de Sentimentos - Score

Dessa forma, observa-se que a ferramenta e metodologia adotadas são de grande valia para a proposta do estudo.

\section{CONSIDERAÇÕES FINAIS}

Um dos principais objetivos desse projeto piloto é testar a técnica de Análise de Sentimentos no desenvolvimento de uma nova Métrica de Sustentabilidade organizacional.

Como acredita-se, a partir da literatura sobre benefícios da sustentabilidade em ambiente organizacional, a satisfação dos colaboradores pode estar relacionada ao aumento da produtividade. Dessa maneira, a análise de sentimentos tende a ser um método efetivo de avaliação de tal impacto, bem como um indicador para composição da Métrica.

Assim, a construção de indicadores, tais como Análise de Sentimento se faz relevante para a composição e desenvolvimento da nova Métrica no estágio atual do projeto de pesquisa, uma vez que poderá trazer mais informações às tomadas de decisões, bem como aferir de forma mais precisa os benefícios da sustentabilidade.

O presente projeto encontra-se em estágio inicial. Está sendo realizados novos testes e aprimoramentos, tais como novas versões de questionário, além de amostras de diferentes ambientes organizacionais, com base nos resultados preliminares obtidos.

\section{AGRADECIMENTOS}

Agradecemos a Fundação Araucária pelo apoio e patrocínio da presente pesquisa, por meio do Programa Institucional de Bolsas de Iniciação Científica (PIBIC).

\section{REFERÊNCIAS}

[1] Bellen, H. M. Van. Indicadores de Sustentabilidade: uma análise comparativa. Ed. 2. Rio de Janeiro: Editora FGV, 2006.

[2] Callado, A. L C. Modelo de mensuração de sustentabilidade empresarial: uma aplicação em vinícolas localizadas na Serra Gaúcha. Porto Alegre. Tese de Doutorado. Universidade Federal do Rio Grande do Sul. 2010.

[3] Comissão Mundial Sobre o Meio Ambiente e Desenvolvimento. Nosso Futuro Comum. Ed. 1. Rio de Janeiro: Editora da Fundação Getúlio Vargas, 1998.

[4] Da Silva, C. F.; Vessen Junior, M.V. Mineração de Opiniões: Conceitos, Desafios e Aplicações. Londrina. Anais do Simpósio Brasileiro de Sistemas de Informação Minicursos. v. 7. p. 7-9. 2014.

[5] De Freitas, M.; Da Silva, M.C.F.; Marmoz, L. A ilusão da sustentabilidade. Ed 1. Manaus: EDUA, 2003

[6] Gama, M. C. F. P. Medidas de bem-estar e sustentabilidade: desenvolvimento de metodologias de análise e seleção de métricas. Lisboa. Universidade Nova. Dissertação de mestrado. 2010.

[7] Gil, A. C. Como elaborar projetos de pesquisa. Ed. 4. São Paulo: Atlas, 2002.

[8] Gouveia, J.M.C. A Métrica da Sustentabilidade na perspectiva da Geografia: aplicação e avaliação do Painel da Sustentabilidade (Dashboard of Sustainability) na Comunidade Quilombola de Mandira. Cananéia. Universidade de São Paulo. Tese de Doutorado. 2010.

[9] Leff, E. Epistemologia Ambiental. Tradução de Sandra Valenzuela. Ed. 4. São Paulo: Cortez, 2007.

[10] Liu, B. (2010) “Sentiment analysis and subjectivity” In: Handbook of natural language processing, 2:568.

[11] Liu, B. (2012) "Sentiment analysis and opinion mining" Morgan \& Claypool Publishers.

[12] Mascarenhas, S. A. Metodologia científica. Ed. 1. São Paulo: Pearson Education do Brasil, 2012.

[13] Pang, B.; Lee, L. (2008) “Opinion Mining and Sentiment Analysis" In: Foundations and Trends in Information Retrieval 2(1-2), pp. 1-135.

[14] Tsytsarau, M.; Palpanas, T. (2010) "Survey on mining subjective data on the web" In: Data Mining and Knowledge Discovery, 24(3):478-514.

[15] Shmilovici, A. Data Mining and Knowledge Discovery Handbook. Ed. 2. Israel. 2005.

[16] Veiga, J. E. Indicadores de sustentabilidade. São Paulo. Estudos avançados. Universidade de São Paulo. v. 24, n. 68, p. 39-52, 2010. Disponível em:

$<$ http://www.scielo.br/pdf/ea/v24n68/06.pdf > . Acesso em: 19 de set. 2014.

[17] Willard, Bob. Como fazer a empresa lucrar com sustentabilidade: aumente a receita e a produtividade e reduza riscos e despesas. Tradução por Cristina Yamagami. Ed. 1. São Paulo: Saraiva 2014. 